\title{
Configuración de un servicio de correos electrónicos institucionales para el Cuerpo de Agentes de Control Metropolitano de Quito
}

fecha de recepción: 24-02-2021 • Fecha de aceptación: 19-04-2021 • Fecha de publicación: 10-06-2021

Luis Ricardo Jácome lema

Cuerpo de Agentes de Control Metropolitano de Quito, Ecuador

ricardolrjl@gmail.com

https://orcid.org/0000-0003-1354-6136

\section{RESUMEN}

El entorno de trabajo hace que cada día se busquen nuevas y mejores formas para enviar y recibir información, el uso de una cuenta de correo electrónico institucional permite mejorar la forma de comunicación entre el personal de esta entidad municipal, por lo que la presente investigación apunta a atender estas necesidades. El Cuerpo de Agentes de Control Metropolitano de Quito (CACMQ) es una institución pública adscrita al Municipio de Quito que cuenta con 1369 servidores municipales. Esta institución no posee un servicio de correos electrónicos como medio de comunicación oficial, por lo que es necesario que se implemente este sistema acorde a la normativa legal vigente establecida para las entidades públicas. En este contexto, este trabajo propone implementar un servicio de correos electrónicos institucionales bajo la metodología EDER, este servicio permitirá que cada servidor del CACMQ utilice una cuenta de correo electrónico en la que puede intercambiar mensajes e información interna y externa con otras entidades públicas y privadas. 


\section{ABSTRACT}

The work environment means that every day new and better ways to send and receive information are sought, the use of an institutional e-mail account allows to improve the form of communication between the staff of this municipal entity, so this research aims to meet these needs. The Corps of Metropolitan Control Agents of Quito (CACMQ) is a public institution attached to the Municipality of Quito and has 1,369 municipal employees. This institution does not have an e-mail service as a means of official communication, so it is necessary to implement this system according to the current legal regulations established for public entities. In this context, this work proposes to implement an institutional e-mail service under the EDER methodology, this service will allow each server of the $C A C M Q$ to use an e-mail account in which they can exchange messages and information internally and externally with other public and private entities.

KEYWORDS: agents, communication, institutional mail, normative. 


\section{Introducción}

El Cuerpo de Agentes de Control Metropolitano de Quito (CACMQ) (Cuerpo de Agentes de Control Metropolitano Quito, 2021) al momento depende de la Dirección Metropolitana de Informática (DMI) para la creación y administración de cuentas de correos electrónicos institucionales, actualmente la institución cuenta aproximadamente con 207 cuentas institucionales, pero el total de servidores municipales que laboran en el CACMQ es de 1350, lo que ha conllevado a que se creen cuentas de correos no institucionales, donde los servidores quieran o no recibir o acatar disposiciones, asumiendo que este medio no es oficial ni autorizado para recibir información.

La DMI menciona que se encuentra con falta de licenciamiento para la creación de cuentas nuevas y existe una limitación en infraestructura actual, lo que no permitiría la creación de correos Electrónicos institucionales para todo el personal del CACMQ.

De igual forma, la institución no cuenta con horarios fijos establecidos, ya que se mantiene una jornada de trabajo especial como se menciona en el Art. 25 de la LOSEP, teniendo turnos especiales de labores, lo que ha provocado que las disposiciones sean emitidas por cuentas de correos electrónicos personales o WhatsApp, medios no oficiales para recibir disposiciones, ocasionando a que no sean cumplidas.

De acuerdo a las Políticas de Tecnología de la Información del Gobierno Autónomo Descentralizado del Distrito Metropolitano de Quito en el apartado 3.7 Política de Uso de Correo Electrónico, Art. 20 Generales: "El correo electrónico institucional es un recurso que el GAD del Distrito Metropolitano de Quito pone a disposición de las y los funcionarios, servidores y trabajadores del GAD DMAQ, como una herramienta de comunicación, colaboración e intercambio de información oficial (...)", por lo que mediante Memorando Nro. GADDMQ-CACMQ-20200100-M, emitido por Director General del Cuerpo de Agentes de Control Metropolitano de Quito, se solicita a la Dirección Metropolitana de Informática (DMI) del Municipio de Quito “(...) la creación de los correos electrónicos para todo el personal del Cuerpo de Agentes de Control Metropolitano Quito (...)". A lo cual mediante memorando Nro. GADDMQ-DMI-2020-00046-M, el Director Metropolitano de Informática indica que; "(...) deberá realizarse con un proyecto específico para el efecto, con presupuesto de la entidad requirente y la DMI estará presta a brindar el apoyo para garantizar la integración y compatibilidad del mencionado servicio con la infraestructura existente en la entidad".

Con lo expuesto, la Unidad de Tecnologías de la Información y Comunicación (TIC) del CACMQ ha decidido mejorar sus procesos, entre ellos se encuentra el medio de comunicación oficial, medio por el cual los funcionarios públicos que laboran en esta entidad envían y reciben información. Es importante establecer los requerimientos para que se implemente este servicio y este acorde a las necesidades demandadas por la institución enmarcadas dentro del ámbito legal.

En este sentido, este trabajo propone implementar un servicio de correos electrónicos institucionales para el Cuerpo de Agentes de Control Metropolitano de Quito, para lo cual se van a desarrollar las siguientes actividades: 
- Levantar los requerimientos necesarios de acuerdo a las directrices y necesidades institucionales.

- Configurar un servidor para correos electrónicos.

- Configurar el Software Zimbra como herramienta Open Source para el servicio de correos institucionales.

- Realizar las pruebas de administración y utilización del sistema de correos electrónicos institucionales del CACMQ.

\section{Metodología}

Según Morales Carrillo \& Cedeño Valarezo (2017) “(...) al no encontrar metodologías para la implementación de infraestructura tecnológica, regularmente se recurre a la adaptación de un modelo. La propuesta de la Metodología EDER (Estudio, diseño, ejecución y revisión) es una alternativa metodológica sencilla y fácil de adaptar a proyectos de infraestructura tecnológica o afines $(. .)$.$" .$

De tal manera, la metodología aplicable a este proyecto es "EDER" (Morales et al., 2018), que contempla cuatro fases: estudio, diseño, ejecución y revisión. Para la aplicación de esta se evidenció la problemática existente en el Cuerpo de Agentes de Control Metropolitano Quito, detectando deficiencias en la infraestructura tecnología, como la falta de servidores funcionales para la implementación y funcionamiento de un servicio de correos electrónicos institucionales.

Cabe mencionar que las metodologías (Rodríguez, 2017) orientan las diferentes fases y actividades que se deben realizar en los proyectos investigativos, determinando claramente lo que debemos realizar, a pesar de aquello y de las múltiples metodologías (Mesh, 2020) agiles aplicadas desarrollo de software, auditorias, entre otras, la más adaptable a este estudio en la mencionada anteriormente, debido a su adaptabilidad.

\subsection{Análisis de la organización}

El Cuerpo de Agentes de Control Metropolitano Quito (CACMQ) es una empresa pública adscrita al Municipio de Quito. De acuerdo a las políticas de tecnología de la información del Gobierno autónomo descentralizado del Distrito Metropolitano de Quito, la Dirección Metropolitana de Informática (DMI) es el ente encargado de crear cuentas de correos electrónicos institucionales a todos los empleados municipales; sin embargo, por falta de infraestructura mediante memorando al CACMQ, se ha indicado que se debe realizar un proyecto para que la institución cuente y administre este servicio.

La Unidad de TIC del CACMQ, como unidad encargada en el ámbito tecnológico a nivel interno, se encuentra a cargo de la implementación de este servicio, mismo que será utilizado por todos los empleados, considerando la normativa legal vigente en temas de adquisición de bienes, 
contratación de servicios e implementación de sistemas informáticos en empresas públicas.

En la siguiente Figura 1 se muestra una representación de la institución.

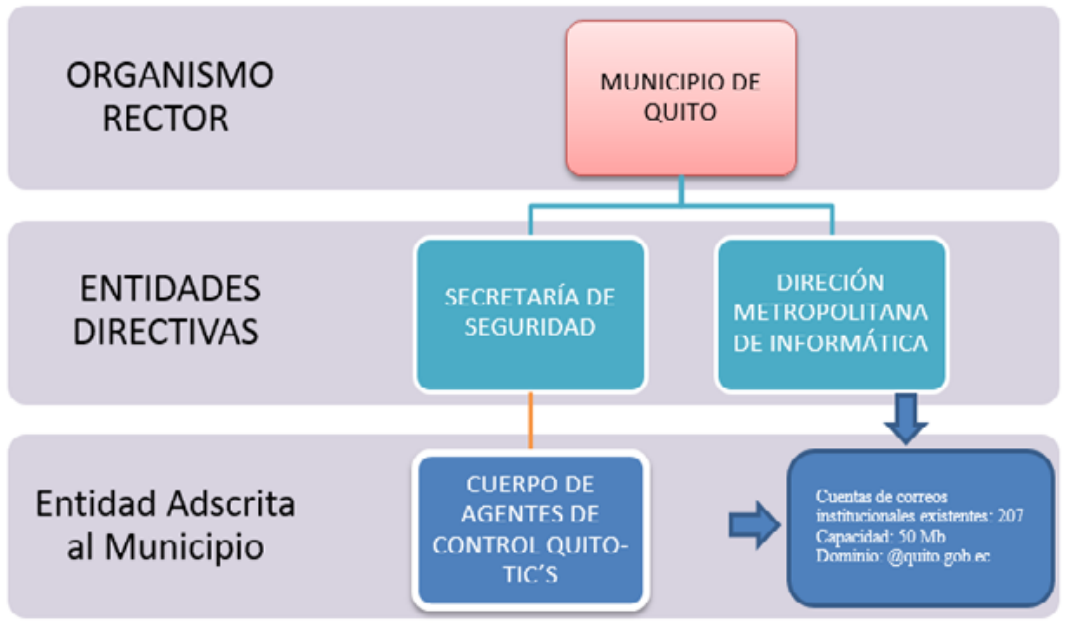

Figura 1. Análisis de la institución

Fuente: elaboración propia

La institución caso de estudio requiere un servicio de correos electrónicos institucionales que gestione la creación de cuentas de correos electrónicos para todo el personal que permita el envío y recepción de información.

Al momento, el número de cuentas institucionales de correos electrónicos debería ser de 1369, pero si consideramos que anualmente ingresan 50 nuevos aspirantes, en 5 años, el número de cuentas de correos electrónicos ascendería a 1619. La DMI ha creado 207 cuentas de correos electrónicos institucionales para los funcionarios del CACMQ, lo cual no satisface a las necesidades requeridas.

A continuación, se presenta en la Tabla 1 un resumen de las cuentas de correos electrónico con una descripción de lo se tiene actualmente, y lo necesario a 5 años, entre lo funcional y lo óptimo. 
Tabla 1.

Resumen de Cuentas de Correos Electrónicos del CACMQ

\begin{tabular}{|c|c|c|c|c|c|}
\hline \multirow[t]{2}{*}{ Detalle } & \multirow{2}{*}{$\begin{array}{l}\text { ACTUAL NO } \\
\text { FUNCIONAL } \\
\begin{array}{l}\text { DMI-Cuentas } \\
\text { creadas }\end{array}\end{array}$} & \multicolumn{2}{|l|}{ FUNCIONAL } & \multicolumn{2}{|l|}{ ÓPTIMO } \\
\hline & & $\begin{array}{l}\text { NECESIDAD } \\
\text { ACTUAL }\end{array}$ & 5 AÑOS & $\begin{array}{l}\text { NECESIDAD } \\
\text { ACTUAL }\end{array}$ & 5 AÑOS \\
\hline Cuentas & 207 & 1369 & 1619 & 1369 & 1619 \\
\hline Megabytes & $50 \mathrm{Mb} /$ cada cuenta & $\begin{array}{l}5000 \mathrm{Mb} / \text { cada } \\
\text { cuenta }\end{array}$ & $\begin{array}{l}5000 \mathrm{Mb} / \text { cada } \\
\text { cuenta }\end{array}$ & $\begin{array}{l}10000 \mathrm{Mb} / \\
\text { cada cuenta }\end{array}$ & $\begin{array}{l}10000 \mathrm{Mb} / \\
\text { cada cuenta }\end{array}$ \\
\hline TOTAL & $\begin{array}{l}12,500 \mathrm{Mb} \\
2.5 \mathrm{~Gb}\end{array}$ & $\begin{array}{l}\text { 6’845,000 Mb } \\
6,845 \mathrm{~Gb} \\
6.845 \mathrm{~Tb}\end{array}$ & $\begin{array}{l}\text { 8'095,000 Mb } \\
8,095 \mathrm{~Gb} \\
8.095 \mathrm{~Tb}\end{array}$ & $\begin{array}{l}13 \text { '690,000 Mb } \\
13,690 \mathrm{~Gb} \\
13.69 \mathrm{~Tb}\end{array}$ & $\begin{array}{l}16{ }^{\prime} 190,000 \mathrm{Mb} \\
16,190 \mathrm{~Gb} \\
16.19 \mathrm{~Tb}\end{array}$ \\
\hline
\end{tabular}

Fuente: elaboración propia

Existe muy poco espacio en las cuentas actuales, cada funcionario tiene 50Mb (Figura 2) para el almacenamiento de correos electrónicos, realizando el análisis respectivo se concluye que lo óptimo sería que cada empleado tenga un espacio en almacenamiento de $10 \mathrm{~Gb}$, lo que significaría que, para un total de 1619 empleados, se necesitaría un espacio de almacenamiento aproximado de $16 \mathrm{~Tb}$.

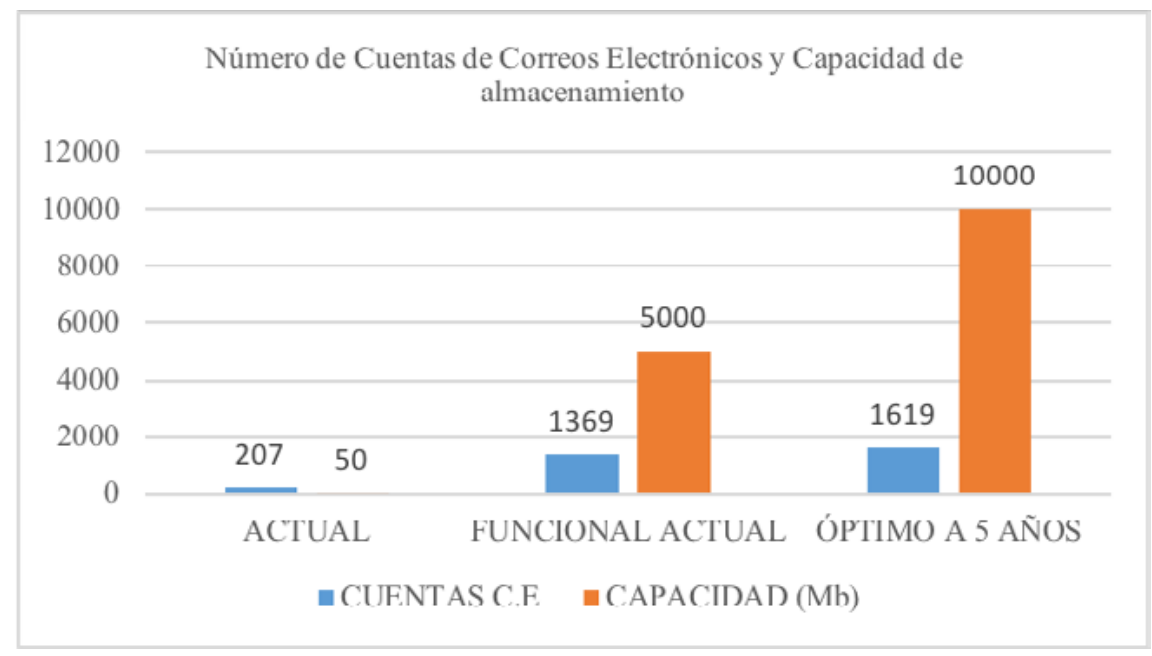

Figura 2. Cuentas de correos electrónicos y capacidad de almacenamiento

Fuente: elaboración propia

Con la implementación del servicio habría un incremento del 648\% en el número de cuentas existentes de correos y con respecto al espacio asignado al buzón de correos, con 5 Gb por cuenta, se mejoraría en un 10,000\% funcionalmente; sin embrago, si las cuentas tuvieran 10Gb de espacio, habrá una mejora del $20,000 \%$.

\subsection{Diseño}




\section{- Hardware}

Servidor de pruebas. - El que se utilizó para el servicio de correos electrónicos tiene las siguientes características generales:

- Procesador: Intel(R) Xeon(R) CPU E31220 @ 3.10GHz, 4 cores

- RAM: 4GB

- Almacenamiento: $250 \mathrm{~Gb}$

- Software

- Centos

Se podrá utilizar CENTOS (Membrey et al., 2009) debido a que es un sistema operativo Open Source, de tipo Unix con código abierto basado en el kernel de Linux cuya versión a utilizar puede ser la 7, debido a que el soporte técnico del sistema en esta versión es hasta el año 2024; sin embargo, se puede utilizar la versión 8 para el ámbito de pruebas, ya que de acuerdo a su página oficial se desconoce aún el año máximo de soporte.

\section{- Webmin}

De manera opcional, pero si se requiere tener un mejor manejo del servidor, de manera remota y mediante web, se ha decidido utilizar la herramienta Webmin (2021), que mediante su página oficial menciona que, "(...) es una interfaz basada en web para la administración de sistemas para Unix. (...) y le permite administrar un sistema desde la consola o de forma remota".

- Zimbra

Utilizaremos Zimbra (s.f) como herramienta gestionadora de correos electrónicos con su última versión estable Open Source 8.8.15, como indican “(...) es una plataforma de mensajería y colaboración que permite enviar y recibir correo electrónico, agendar citas y reuniones, crear contactos, organizar tareas y compartir documentos y archivos (...)".

\section{- Apache JMater}

Utilizaremos Apache JMeter (s.f), herramienta para realizar las pruebas de estrés del servidor de correos, que de acuerdo a su sitio web esta "es un software de código abierto, una aplicación Java $100 \%$ pura diseñada para cargar el comportamiento funcional de prueba y medir el rendimiento (...)".

- Putty

Para realizar la conexión SSH del usuario administrador con el servidor se lo hará mediante la herramienta Putty, con el fin de verificar y realizar configuraciones mediante la consola de 
comandos. "PuTTY es un emulador gratuito de terminal que soporta SSH y muchos otros

protocolos. (...) lo encuentran muy útil a la hora de conectar a un servidor Unix o Linux a través de SSH" (Actualidad.es, 2020).

A su vez, se considera que la IP debe ser pública, de acuerdo a lo solicitado a CNT por el Cuerpo de Agentes de Control.

En cuanto al dominio, el que se va a utilizar para la etapa de pruebas es uno gratuito adquirido en la Plataforma Freenom (s.f), el primer proveedor de dominios gratuitos del mundo.

Para la fase de producción se deberá contratar un dominio de tercer nivel (dominio.gob.ec), mediante un proceso de contratación pública.

Para la etapa de pruebas se puede hacer con un certificado gratuito adquirido en la Autoridad Certificadora Let's Encrypt (s.f), misma que menciona en su página oficial "(...) es una autoridad de certificación gratuita, automatizada, y abierta traída a ustedes por la organización sin ánimos de lucro".

Para la fase de producción se deberá contratar un certificado SSL, mediante un proceso de contratación pública.

\subsection{Ejecución}

La siguiente Figura 3 se muestra como se realizó la instalación de Centos en modo consola.

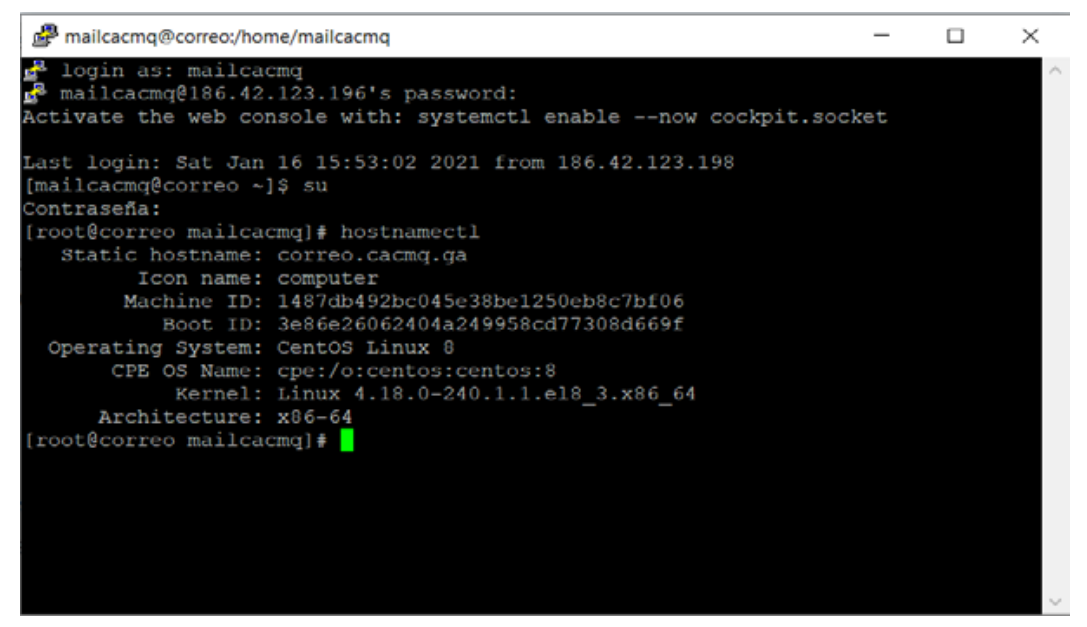

Figura 3. Instalación de Centos

Fuente: elaboración propia

En la siguiente Figura 4 se observa la instalación de Webmin para la administración y verificación del servidor. 


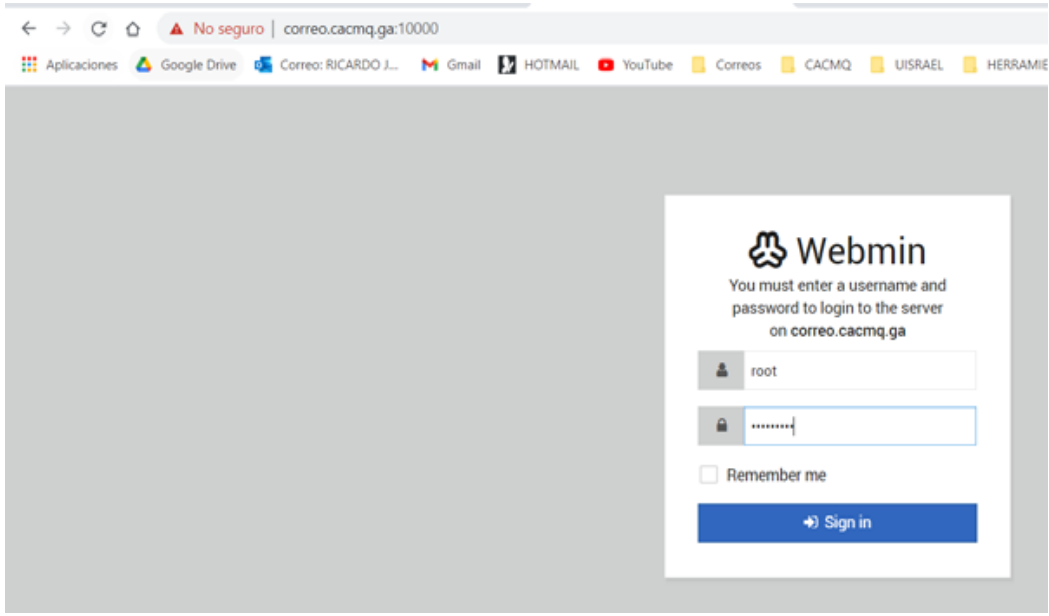

Figura 4. Instalación de Webmin

Fuente: elaboración propia

Puertos abiertos del Firewall del Servidor que se utilizaron fueron: 25, 80, 110, , 143, 443, 465, 587, 993, 3443, 9071, 7071.

La configuración del dominio fue DNS (A y MX), tal como se ve en la Figura 5.

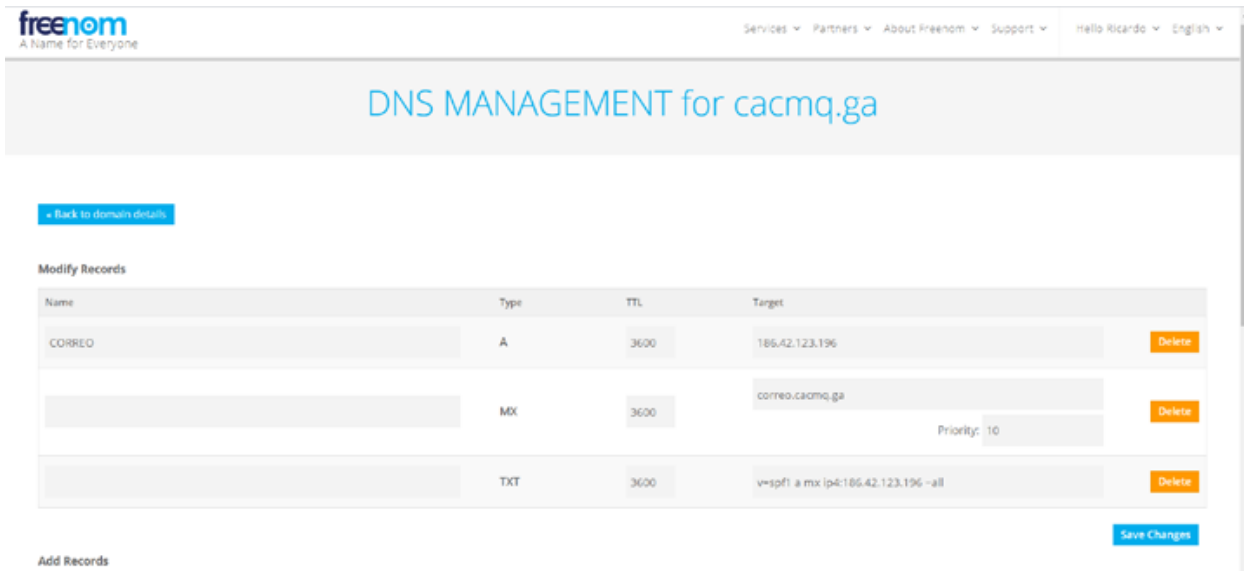

Figura 5. Configuración del dominio

Fuente: elaboración propia

La instalación de Zimbra 8.8.15 se observa en la Figura 6. 


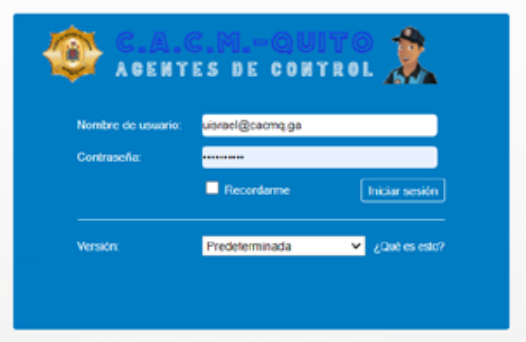

Figura 6. Instalación de Zimbra

Fuente: elaboración propia

Mientras que la configuración de Apache JMeter con pruebas de estrés y concurrencia de usuarios se ve la siguiente Figura 7.

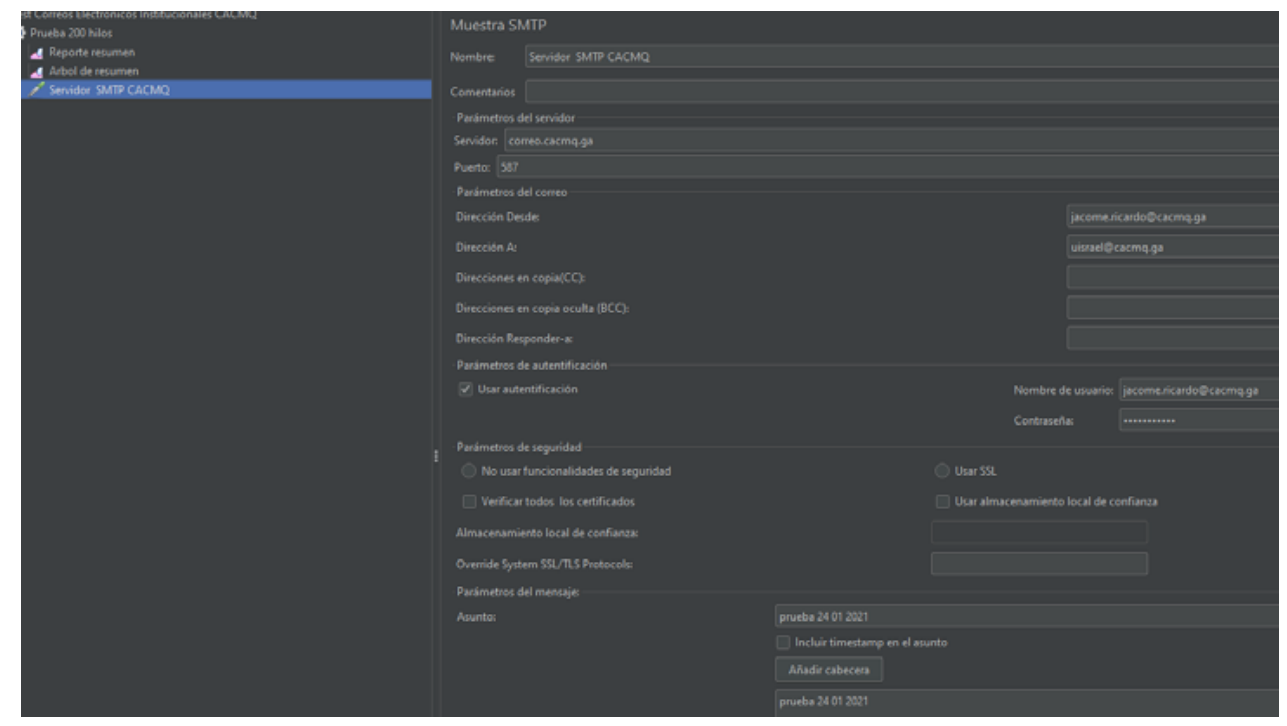

Figura 7. Configuración de Apache JMeter

Fuente: elaboración propia

En cuanto a la revisión, se muestran las siguientes representaciones. Estas se realizaron en la página IPDatabase.com

En la siguiente Figura 8 se muestra la verificación de la IP. 


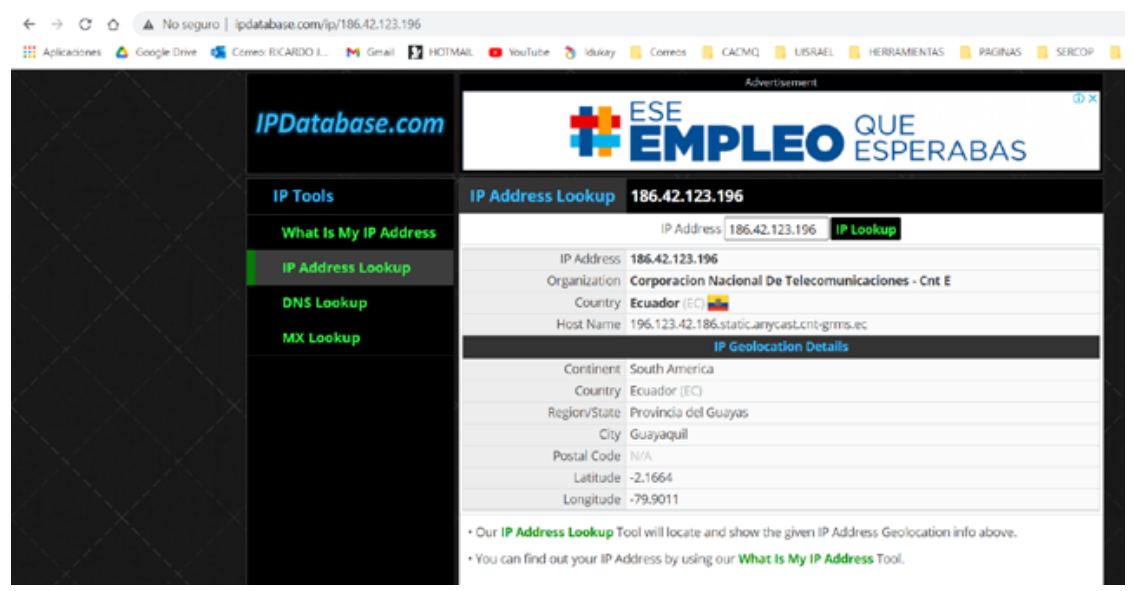

Figura 8. Verificación de la IP

Fuente: elaboración propia

A su vez, la verificación del DNS Address se muestra en la Figura 9.

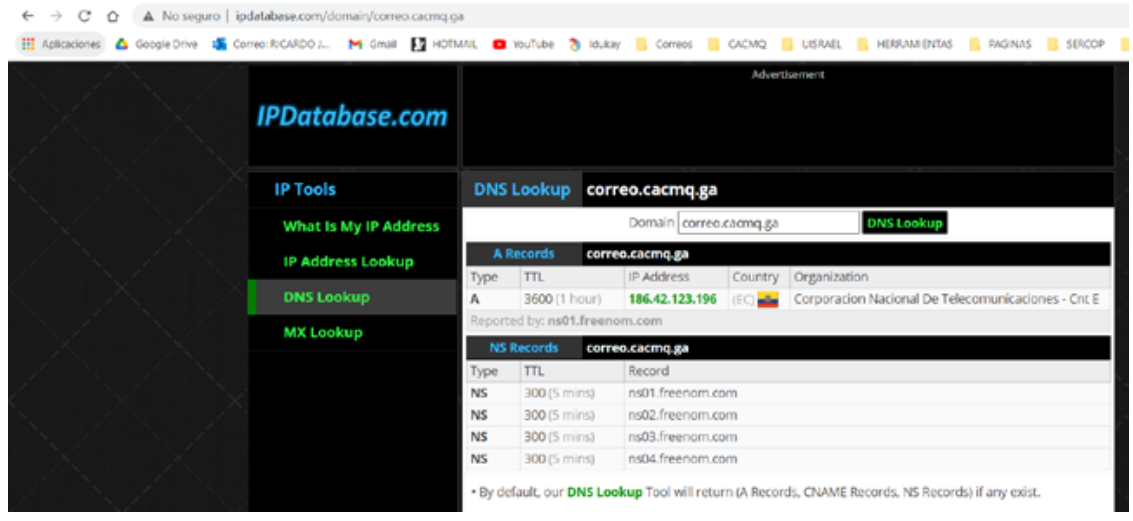

Figura 9. Verificación del DNS Address

Fuente: elaboración propia

En cuanto a la verificación del DNS MX, se muestra a continuación en la Figura 10.

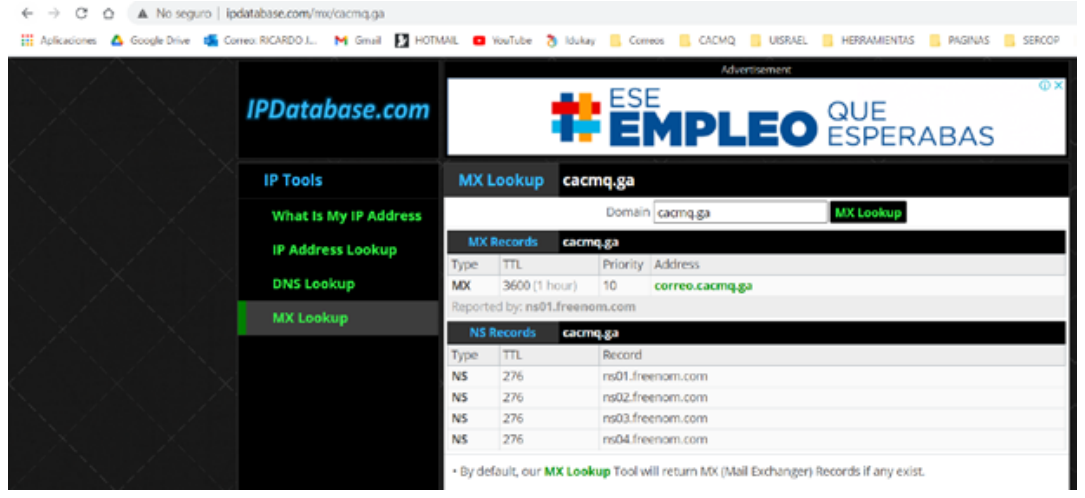

Figura 10. Verificación del DNS MX

Fuente: elaboración propia 
En la Figura 11 se muestra la verificación de los puertos abiertos en el Firewall del servidor. Mediante Webmin se puede evidenciar qué puertos se encuentran abiertos para conectarse con los servicios.

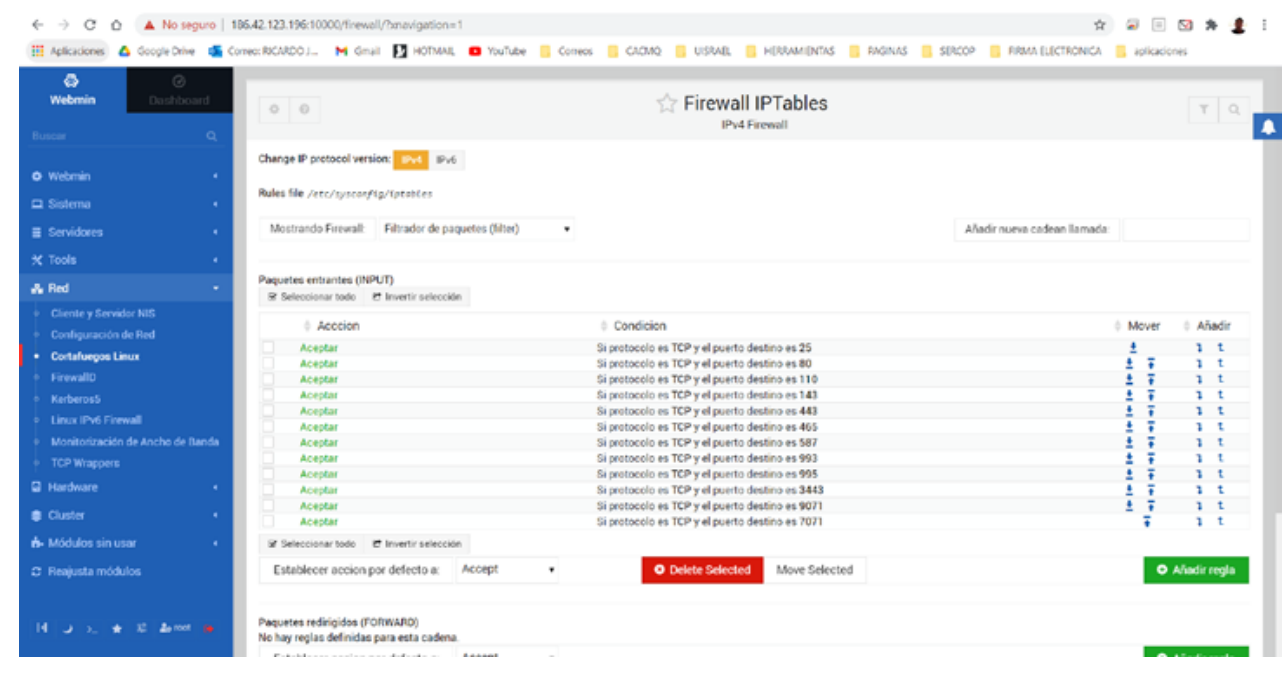

Figura 11. Verificación de los puertos

Fuente: elaboración propia

La verificación de estado de los servicios del Sistema Zimbra se ejecutaron normalmente, como se observa en la Figura 12.

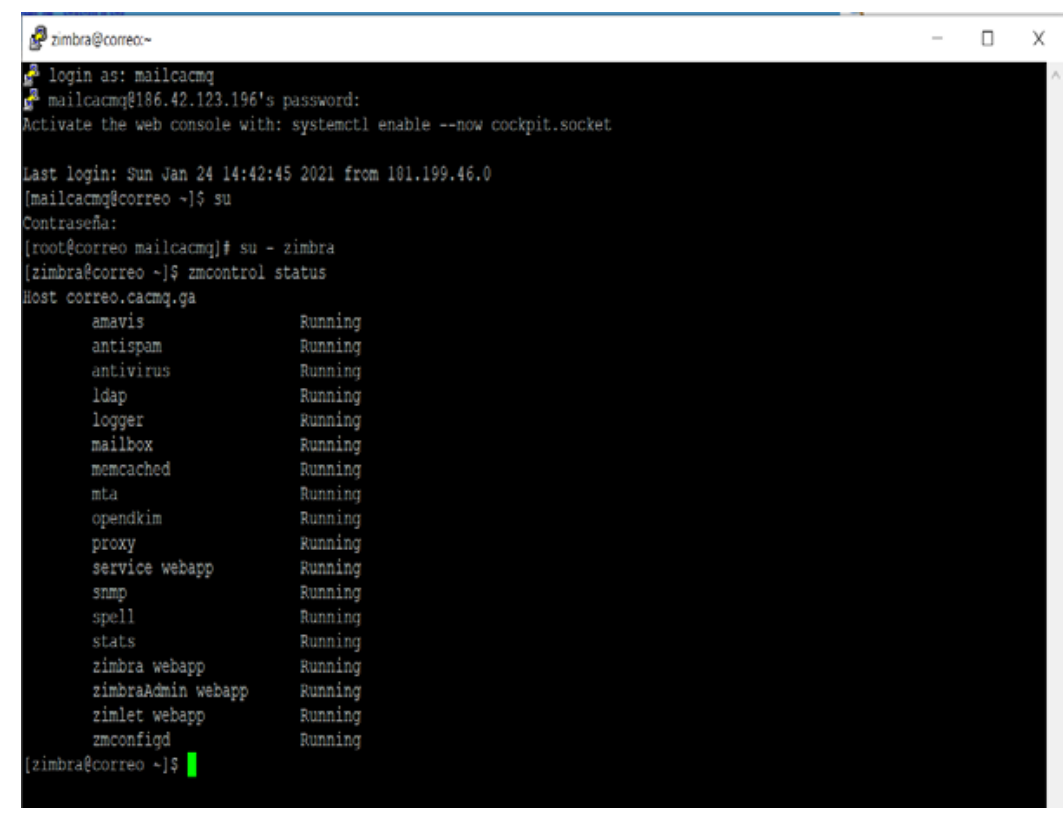

Figura 12. Verificación de estado de los servicios de Zimbra

Fuente: elaboración propia 
Se realizó el envío de correo a una cuenta externa (Figura 13).

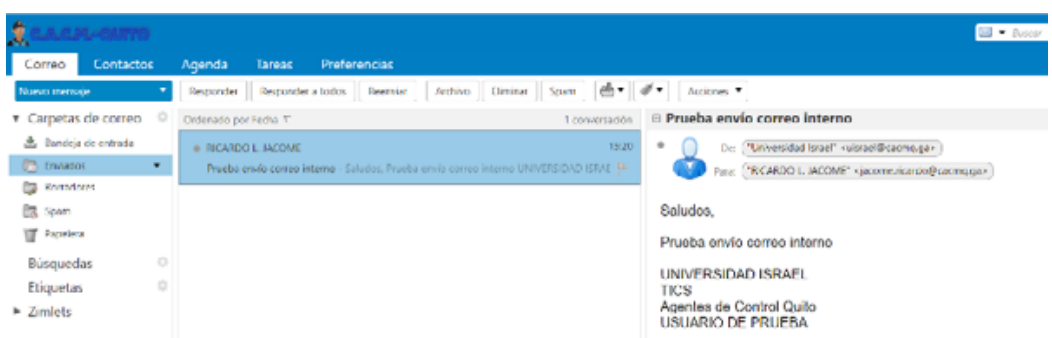

Figura 13. Envío de correo

Fuente: elaboración propia

Y se evidenció como sería la recepción de este mediante Gmail (Figura 14).

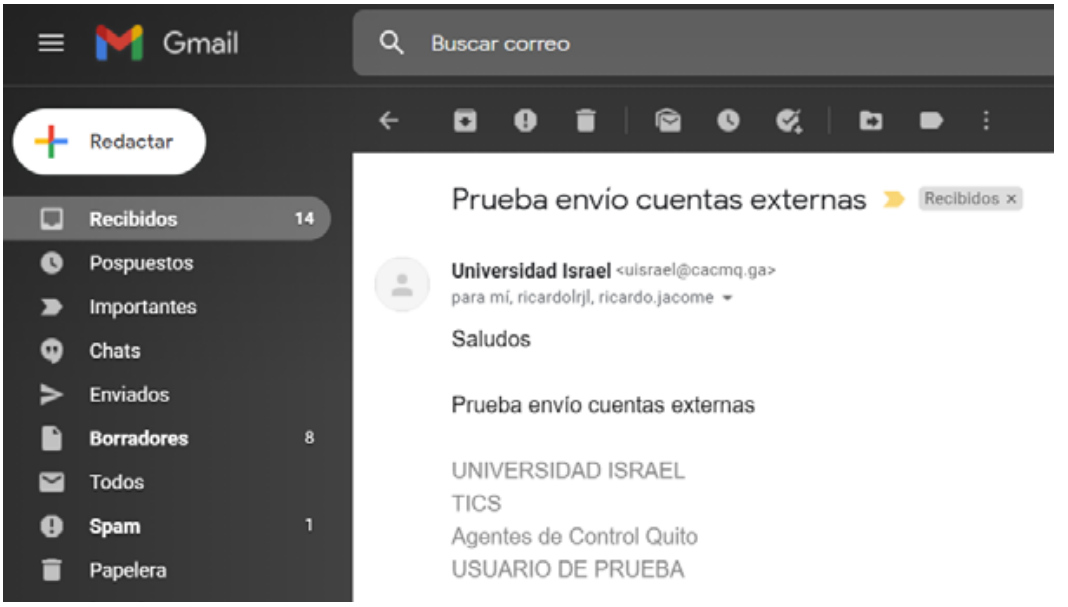

Figura 14. Recepción de correo

Fuente: elaboración propia

Además, se registró que en el chat institucional (Figura 15) este se encuentra funcional y se puede incluir más usuarios del sistema. 


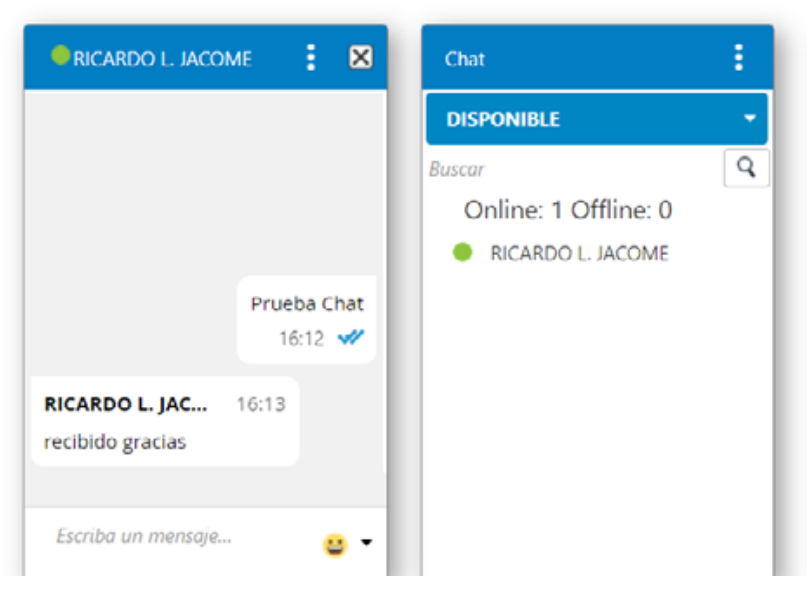

Figura 15. Chat institucional

Fuente: elaboración propia

\section{Resultados}

De acuerdo a la Figura 16, se evidencia que la memoria de nuestro servidor es de 4 GB de RAM, se muestra en estado rojo y al $86 \%$ en uso, por lo que el servidor está usando la memoria virtual, lo cual no es muy recomendable. Cabe añadir que al momento se tienen 35 cuentas creadas para la etapa de prueba realizada con usuarios de la Central de Radio y Telecomunicaciones del CACMQ. Por tal motivo es necesario que el servidor que se vaya adquirir para la etapa de producción tenga una memoria RAM de mayor capacidad para un mejor rendimiento.

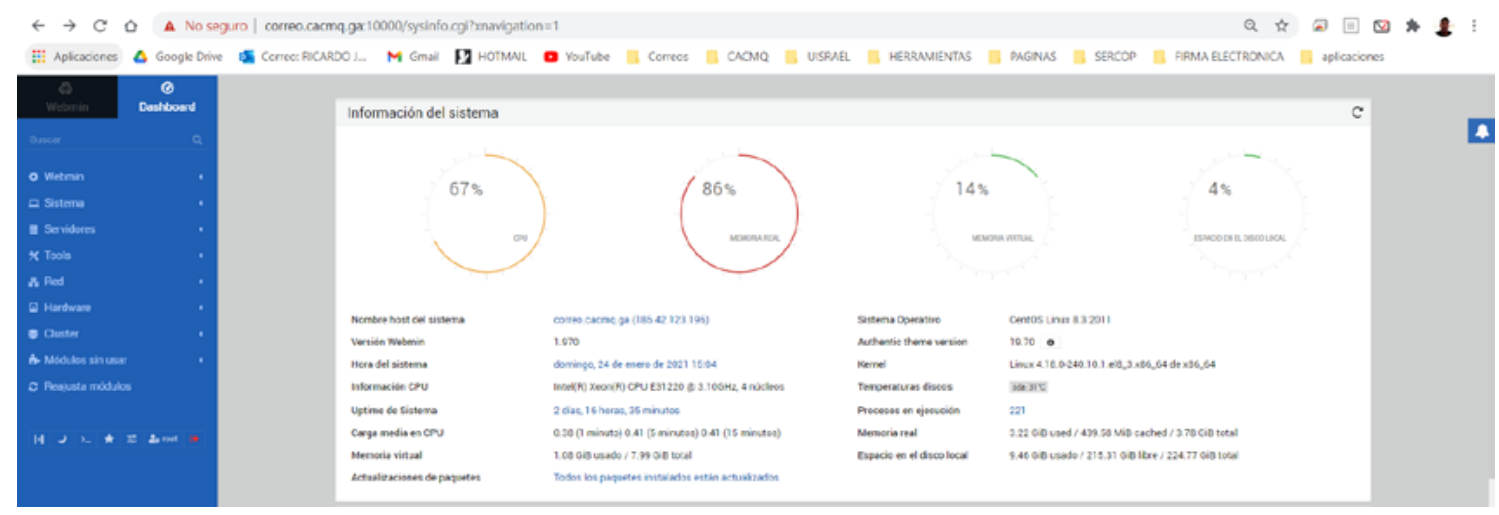

Figura 16. Estado del servidor desde Webmin

Fuente: elaboración propia

Los registros DNS del dominio ADDRESS (A) apuntan correctamente a nuestra IP publica y el registro Mail exchange record (MX) del dominio cacmq.ga apunta correctamente al host del servidor correo.cacmq.ga. Por lo cual no hay conflicto en el dominio con el servidor y el servicio funciona correctamente, tal como se evidencia en la Figura 17. 


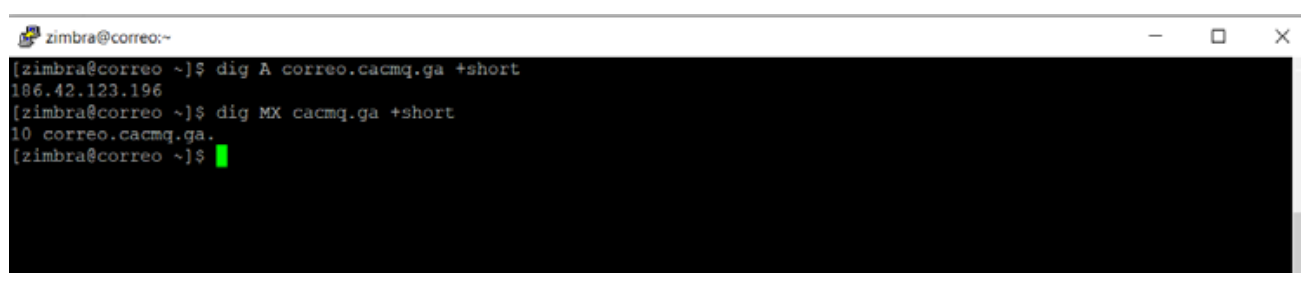

Figura 17. Estado de los DNS del Dominio

Fuente: elaboración propia

El certificado configurado (Figura 18) tiene una fecha de validez desde el 02 de enero del 2021 y una fecha de caducidad del 02 de abril del 2021.

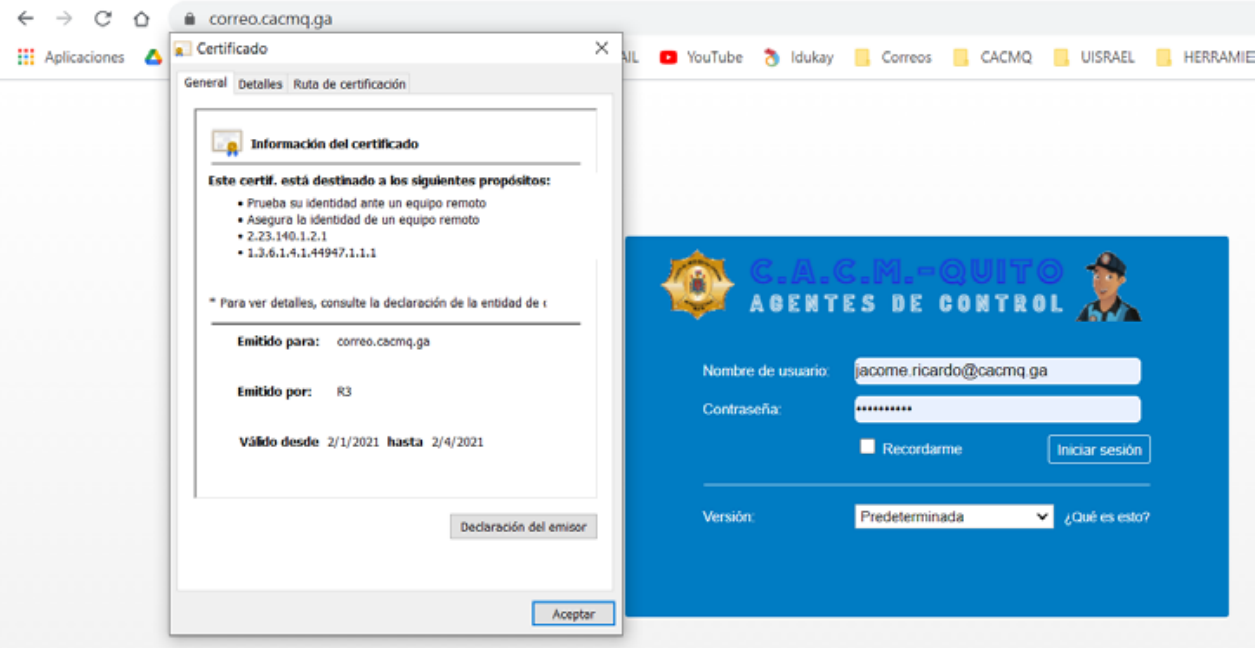

Figura 18. Certificado de Seguridad SSL

Fuente: elaboración propia

En la siguiente Figura 19 se puede ver la prueba 1: Concurrencia de 200 Hilos en Apache JMeter.

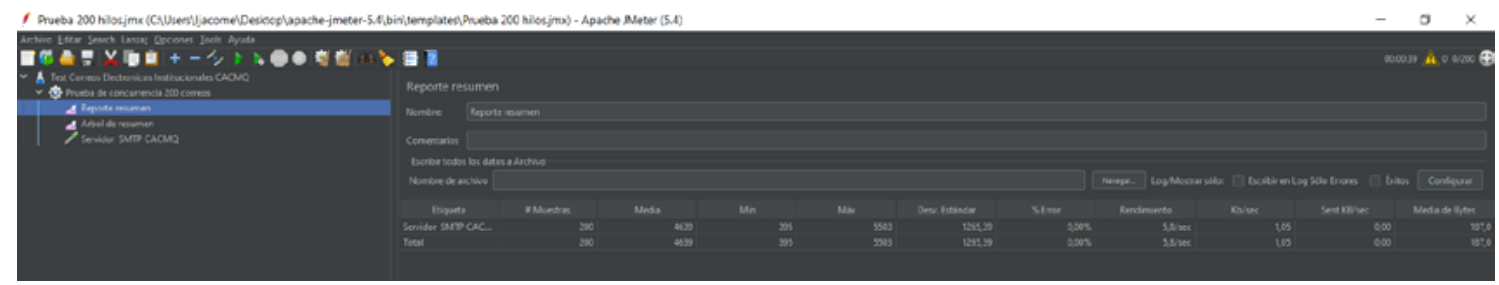

Figura 19. JMeter-Prueba de Concurrencia de 200 Hilos

Fuente: elaboración propia

Se entregaron 200 correos en una duración de 3 minutos de los 200 enviados, no hubo errores (Figura 20). 


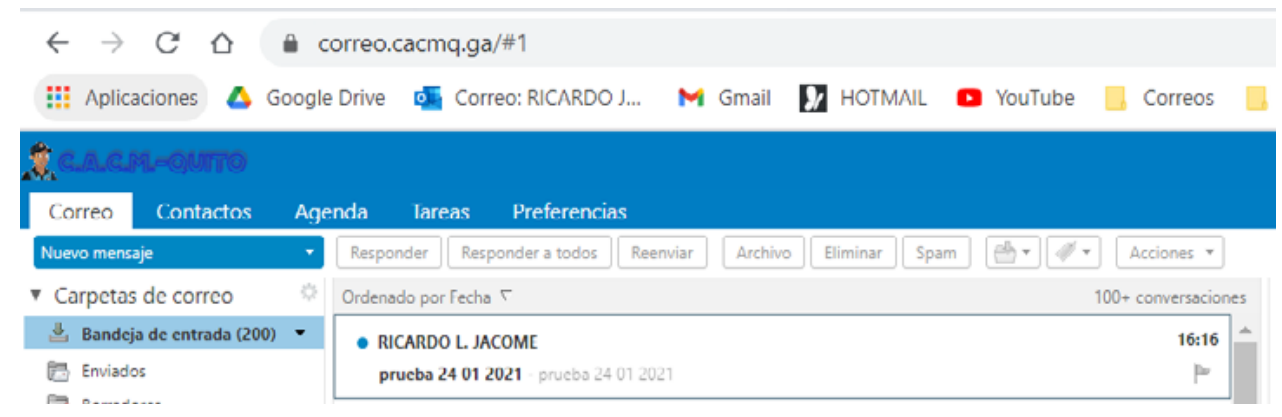

Figura 20. Sistema Zimbra- Prueba de 200 hilos

Fuente: elaboración propia

En la Figura 21 se observa la prueba 2: Concurrencia de 400 correos electrónicos en Apache JMeter.

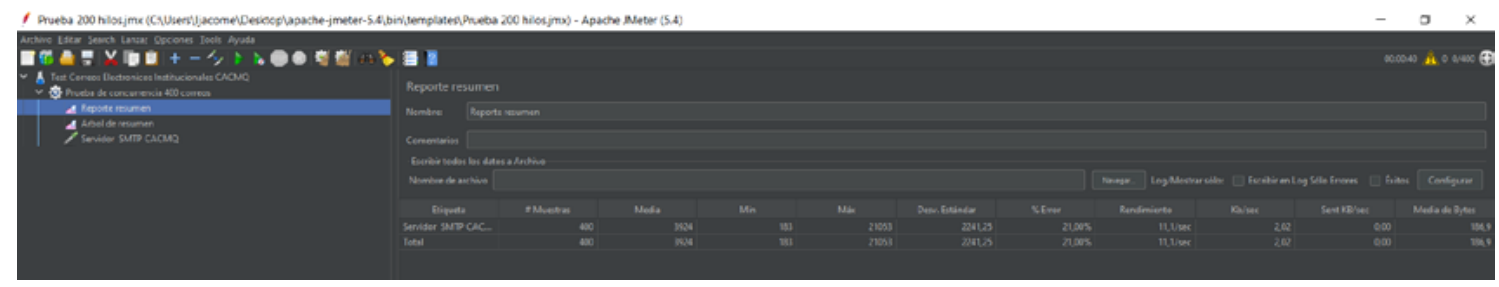

Figura 21. JMeter-Prueba de Concurrencia de 400 Hilos

Fuente: elaboración propia

Se entregaron 316 correos en una duración de 3 minutos de los 400 enviados, con un error del $21 \%$ (Figura 22).

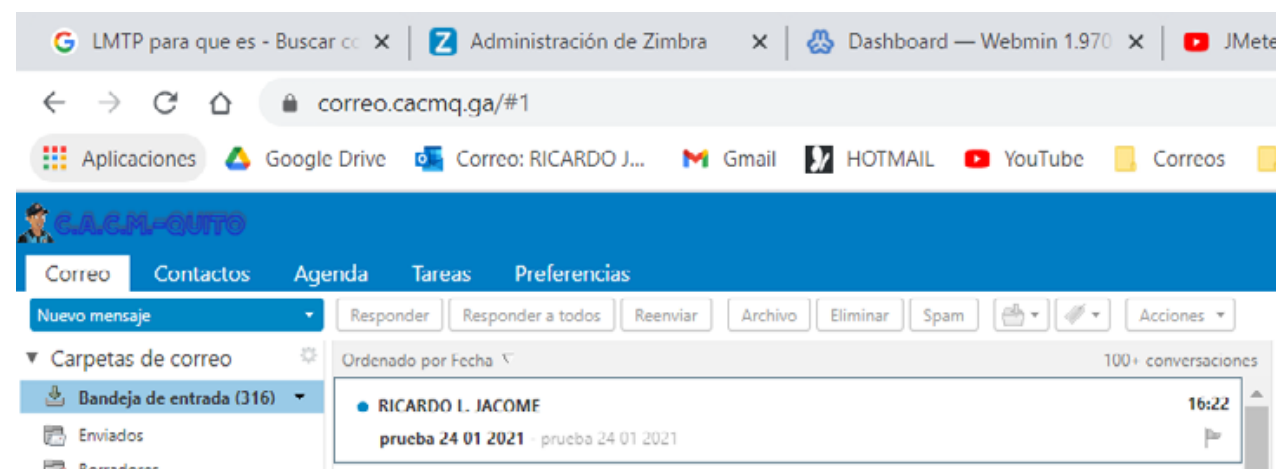

Figura 22. Sistema Zimbra- Prueba de 400 hilos

Fuente: elaboración propia

La prueba 3: Concurrencia de 600 correos electrónicos en Apache JMeter se muestra en la Figura 23. 


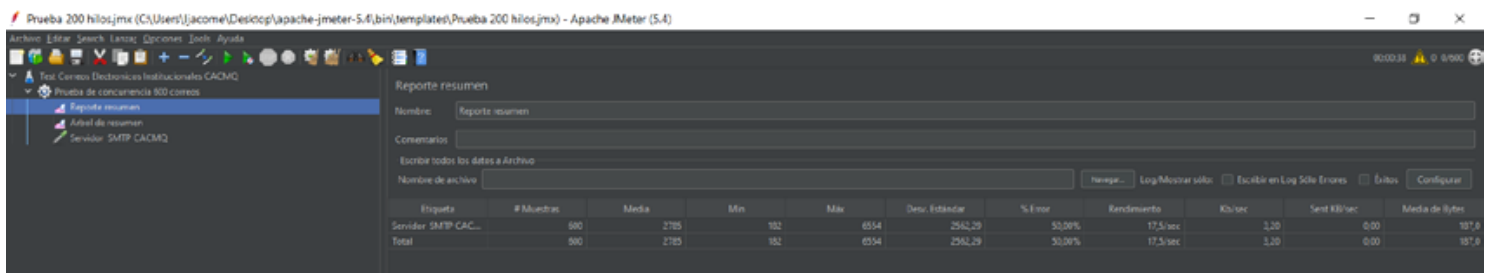

Figura 23. JMeter-Prueba de Concurrencia de 600 Hilos

Fuente: elaboración propia

Se entregaron 300 correos en una duración de 3 minutos de los 600 enviados, con un error del $50 \%$ (Figura 24).

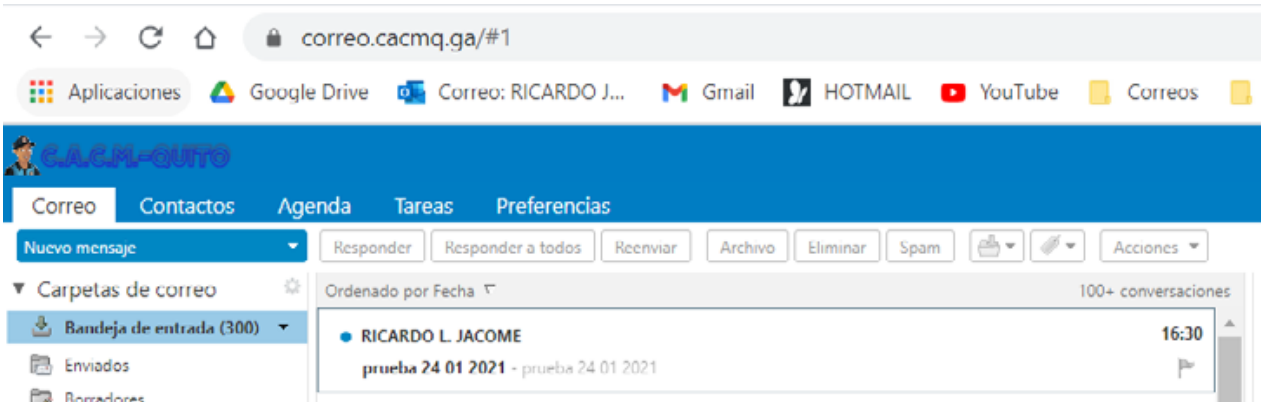

Figura 24. Sistema Zimbra- Prueba de 600 hilos

Fuente: elaboración propia

En la Figura 25 se muestra cómo fue la prueba 4: concurrencia de 1000 correos electrónicos en Apache JMeter

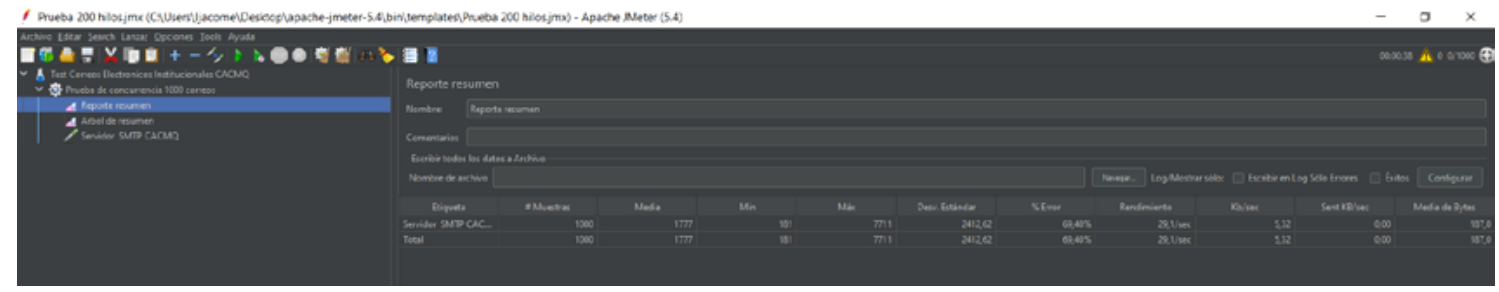

Figura 25. JMeter-Prueba de Concurrencia de 1000 Hilos

Fuente: elaboración propia

Como se puede ver en la Figura 26, se entregaron 306 correos en una duración de 3 minutos de los 1000 enviados, con un error del $69.4 \%$. 


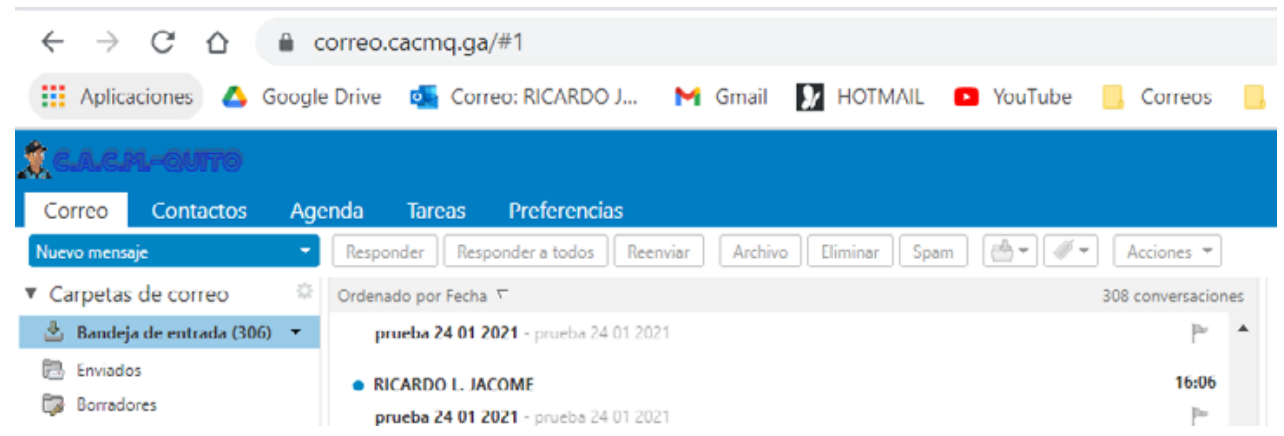

Figura 26. Sistema Zimbra- Prueba de 1000 hilos

Fuente: elaboración propia

En la siguiente Figura 27 se muestran los resultados de las pruebas realizadas en Apache JMeter

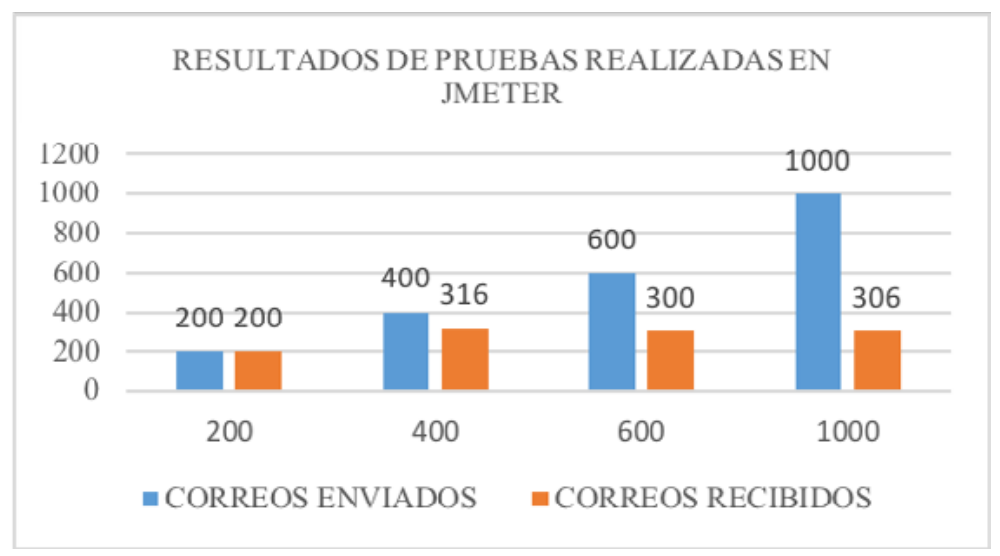

Figura 27. Resultados de las Pruebas realizadas en JMeter

Fuente: elaboración propia

Hay que considerar varios aspectos a la hora de levantar el servicio de correos electrónicos tales como la conexión a internet, concurrencia, especificaciones técnicas del servidor entre otras. Con los resultados obtenidos podemos concluir que de acuerdo a las recomendaciones de "Zimbra Collaborations" para una buena gestión del servicio con 200 usuarios concurrentes se debe trabajar con una memoria RAM mínima de 4 GB e ir incrementándola de acuerdo al número de usuarios. Según las pruebas realizadas es correcto, ya que con una concurrencia de 200 usuarios no hubo errores, pero con la concurrencia de 400, 600 y 1000 únicamente se pudieron enviar hasta un aproximado de 300 correos electrónicos sin error, por lo cual se debería aumentar la memoria al servidor, ya que este es un factor importante para brindar un buen servicio.

\section{Conclusiones}

El proceso de levantar los requerimientos permitió conocer la problemática y necesidades actuales en lo referente a la comunicación, estableciendo que el servicio de correos institucionales es uno de los medios legales para emitir y recibir información institucional. 
La correcta configuración del servidor y de las herramientas instaladas permitió la implementación del servicio de correos electrónicos institucionales en su fase de pruebas; sin embargo, para la etapa de producción las especificaciones técnicas del servidor deben cumplir otros parámetros especificados en el documento.

La plataforma "Zimbra" en la versión 8.8.15, última estable, cumple con las necesidades y requerimientos del CACMQ como herramienta oficial para el intercambio de información institucional e interinstitucional mediante el uso correos electrónicos.

Se concluye a su vez que la utilización del software gratuito contribuye en la automatización de procesos enmarcándonos en la normativa legal vigente para instituciones públicas, reduciendo los costos sin afectar el rendimiento, adaptándose a las necesidades, por lo cual el uso de este software no es un limitante. 


\section{Referencias}

Actualidad.es. (29 de diciembre de 2020). ¿Qué es PUTTY y para qué sirve? Ciencia y Tecnología. https://www. actualidad.es/ciencia-y-tecnologia/2020/04/04/que-es-putty-y-para-que-sirve/

Cuerpo de Agentes de Control Metropolitano Quito. (2021). https://cuerpodeagentesdecontrolquito.gob.ec/

Encrypt, L. (s.f.). Let’s Encrypt. https://letsencrypt.org/es/

Freenom. (s.f.). Empresa. https://www.freenom.com/es/aboutfreenom.html

JMeter, A. (s.f.). Apache JMeter. Obtenido de https://jmeter.apache.org/

Mesh, J. (29 de marzo de 2020). Metodología Kanban: revoluciona tu manera de trabajar más ágil. Blog Trello. https://blog.trello.com/es/metodologia-kanban

Membrey, P., Verhoeven, T., Angenendt, R., de Bruijn, B., \& Singh, K. (2009). The definitive guide to CentOS. Apress. https://doi.org/10.1007/978-1-4302-1931-6

Morales Carrillo, J. J., \& Cedeño Valarezo, L. C. (2017). Propuesta de una metodología para el desarrollo de proyectos de infraestructura tecnológica. Manabi.

Morales, J. J., Cedeño, L. C., Parraga-Alava, J. A., \& Molina, B. A. (2018). Propuesta Metodológica para Proyectos de Infraestructura Tecnológica en Trabajos de Titulación. Información Tecnológica, 29(4), 249-258. https://doi.org/10.4067/S0718-07642018000400249

Rodríguez, M. (2017). Scrum desde cero. Madrid: Mc. Graw-Hill.

Webmin. (2021). What is Webmin? https://www.webmin.com/

Zimbra. (s.f.). ¿Qué es zimbra? Zimbra support. https://www.zimbra-support.net/index.php/es/

Zimbra. (2020). System Requirements for Zimbra Collaboration. https://zimbra.github.io/installguides/latest/single.html\#System Requirements 
Copyright (c) 2021 Luis Ricardo Jácome lema

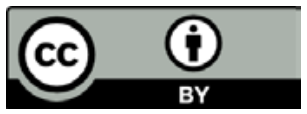

Este texto está protegido bajo una licencia internacional Creative Commons 4.0.

Usted es libre para Compartir-copiar y redistribuir el material en cualquier medio o formato - y Adaptar el documento - remezclar, transformar y crear a partir del material-para cualquier propósito, incluso para fines comerciales, siempre que cumpla las condiciones de Atribución. Usted debe dar crédito a la obra original de manera adecuada, proporcionar un enlace a la licencia, e indicar si se han realizado cambios. Puede hacerlo en cualquier forma razonable, pero no de forma tal que sugiera que tiene el apoyo del licenciante o lo recibe por el uso que hace de la obra.

Resumen de licencia - Texto completo de la licencia 